\title{
Neutrophil Adherence to Human Endothelium In Vitro Occurs by CDw18 (Mo1, MAC-1/LFA-1/GP 150,95) Glycoprotein-dependent and -independent Mechanisms
}

\author{
G. A. Zimmerman* and T. M. Mcintyre** \\ Nora Eccles Harrison Cardiovascular Research and Training Institute and the Departments of Internal Medicine* \\ and Biochemistry, ${ }^{\ddagger}$ University of Utah School of Medicine, Salt Lake City, Utah 84112
}

\section{Abstract}

Components of the CDw18 leukocyte surface glycoprotein complex (Mo1/LFA-1/GP 150,95 or MAC-1, LFA-1 family) are required for some adhesion-related functions of human neutrophils (PMNs). We evaluated the ability of monoclonal antibodies (MoAb) directed against specific determinants on the CDw18 glycoproteins to inhibit neutrophil adherence to cultured human endothelial cells (EC) stimulated by a variety of agonists, including thrombin and leukotriene $\mathrm{C}_{4}$, which induce the EC-dependent adhesion of PMNs. MoAb 60.3, an antibody that binds to an epitope common to the 3 heterodimer subunits of the neutrophil CDw18 complex, potently inhibited (90-100\%) the rapid (5-30 minute) adherence response stimulated by $\mathbf{N}$-formyl-methionyl-leucyl-phenylalanine, leukotriene $B_{4}$, platelet-activating factor, phorbol myristate acetate, Ionophore A23187, and tumor necrosis factor. MoAbs directed against epitopes on the $\alpha$ polypeptide of the CD11b (Mol, MAC-1) heterodimer also inhibited PMN adherence to EC and to cell-free surfaces induced by these agonists. In contrast, the anti-CDw18 MoAbs had a trivial effect on maximal ECdependent neutrophil adherence stimulated by thrombin and leukotriene $\mathrm{C}_{4}$, and incompletely inhibited PMN adherence induced by these agonists under submaximal conditions. These findings indicate that there is an alternative mechanism for neutrophil adherence, presumably resulting from molecular alterations of the EC surface, that does not require the PMN CDw18 glycoproteins. They also suggest that the inability to adhere to endothelium may not completely account for the defect in chemotaxis that is observed in vivo in neutrophils that are deficient in the CDw18 complex.

\section{Introduction}

The adherence of neutrophils (PMNs, granulocytes) to the endothelium is a key event in acute inflammation. It is required for an effective chemotactic response and also for some forms of neutrophil-mediated vascular injury $(1,2)$. When stimulated by specific agonists the neutrophils (3), the endothelial cells $(\mathrm{EC})^{1}(4,5)$, or both $(6)$, become adhesive, resulting in

Address reprint requests to Dr. Zimmerman, NEH CVRTI, Building 100, University of Utah, Salt Lake City, UT 84112.

Received for publication 26 December 1986 and in revised form 9 September 1987.

1. Abbreviations used in this paper: EC, endothelial cell; fMLP, $N$-for$m y l$-methionyl-leucyl-phenylalanine; Io A23187, calcium ionophore

J. Clin. Invest.

(C) The American Society for Clinical Investigation, Inc.

0021-9738/88/02/0531/07 \$2.00

Volume 81, February 1988, 531-537 accumulation of granulocytes on the EC surface. Recently, a glycoprotein that is involved in adhesive interactions has been identified on the surface of activated human neutrophils and has been designated the CD11b or Mol glycoprotein (7-10). The molecule is one of a family of three heterodimers, each consisting of a common $\beta$ polypeptide chain and a variable $\alpha$-chain, termed the CDw18 complex (1, 7-8). Alternatively the heterodimers have been called the LFA-1, MAC-1, or the Mo1/LFA-1/GP 150,95 glycoprotein family $(9,10)$. The $\mathrm{CDw} 18$ complex is critically involved in a variety of functions of neutrophils and other leukocytes. Studies with antibodies directed against specific epitopes on the glycoproteins (10), and of leukocytes from patients who have an inherited deficiency of the molecules (7-11), have indicated that one or more components of the CDw18 complex is required for effective neutrophil chemotaxis and for other adhesion-related functions. Endothelial cell-dependent neutrophil adhesion stimulated by some agonists (8), as well as increased neutrophil adhesiveness resulting from the action of agonists that directly activate the granulocytes $(7,10)$, appears to be largely mediated by expression of the neutrophil CDw 18 complex.

In previous studies we found that thrombin and the sulfidopeptide leukotriene, $\mathrm{LTC}_{4}$, cause the endothelial cell-dependent adherence of neutrophils to human endothelial monolayers $(4,12)$. In the experiments described in this report we examined the effect of monoclonal antibodies directed against the CDw 18 glycoproteins of PMNs on adhesion stimulated by thrombin and $\mathrm{LTC}_{4}$. The results indicate that the neutrophil adhesive glycoproteins are not absolutely required for adherence to endothelium stimulated by these agonists, in contrast to their requirement in adhesion induced by $\mathrm{N}$-for$m y l$-methionyl-leucyl-phenylalanine (fMLP), leukotriene $\mathbf{B}_{4}$, and a variety of other agonists that directly enhance the adhesiveness of neutrophils.

\section{Methods}

Human endothelial cells. Monolayers of umbilical vein EC were cultured as described (4). Only tightly confluent, primary monolayers of EC were used for these experiments.

Adherence of neutrophils to EC monolayers. The labeling of suspensions of isolated human PMNs with ${ }^{11}$ In oxine and quantitation of the adherence of the radiolabeled granulocytes to endothelial monolayers were done exactly as described (4). We also examined each monolayer by phase contrast microscopy to confirm the values calculated from the binding of ${ }^{11}$ In-labeled neutrophils. Agonists for PMN adherence or control buffer were added to PMN suspensions overlying EC monolayers and incubated for various times as indicated. The concentration of each agonist used to stimulate PMN adhesion, and

A23187; LT, leukotriene; MoAb, monoclonal antibody; PAF, platelet-activating factor; PMA, phorbol myristate acetate; TNF, human tumor necrosis factor $\alpha$. 
the duration of incubation after addition of the agonist, were chosen to yield maximal adherence (based on preliminary studies) except where stated otherwise. These agonists included: fMLP, phorbol myristate acetate (PMA), and calcium ionophore A23187 (IoA23187) from Sigma Chemical Co. (St. Louis, MO); platelet-activating factor (PAF; 1-0-alkyl-2-acetyl-sn-glycero-3-phosphocholine) from Avanti Polar Lipids (Birmingham, $\mathrm{AL}$ ); leukotrienes $\mathrm{B}_{4}\left(\mathrm{LTB}_{4}\right)$ and $\mathrm{C}_{4}\left(\mathrm{LTC}_{4}\right)$, provided by Joshua Rokach, Merck-Frosst Canada Inc. (Quebec, Canada); human $\alpha$ thrombin, provided by George Broze (Washington University, St. Louis, MO) and John Fenton (New York State Department of Health, Albany, NY); and recombinant human tumor necrosis factor (TNF $\alpha$ ), a gift from Michael Shepard, Genentech, Inc. (South San Francisco, CA). In some experiments thrombin or $\mathrm{LTC}_{4}$ was added to the EC for variable periods, the monolayers were washed, and PMNs were added for a subsequent 5-min incubation period $(4,12)$.

Adherence of neutrophils to cell-free surfaces. The adhesion of ${ }^{111}$ In-labeled granulocytes to polystyrene petri dishes preincubated with human serum albumin or with $0.2 \%$ gelatin was measured as described (13).

Monoclonal antibodies. Mouse monoclonal antibodies (MoAb) $60.1,60.3$, and 60.5 were generously provided by John Harlan and Patrick Beatty of the University of Washington and the Fred Hutchinson Cancer Center, Seattle, Washington. MoAb 60.3 is an IgG2a antibody $(6-8,14)$ that precipitates all of the polypeptide chains of the multimeric CDw18 cell surface glycoprotein complex (7), indicating that it is directed against an epitope on the common beta chain or against an epitope defined by the quarternary structure of the molecules $(7,10)$. MoAb 60.1 is an IgG1 antibody directed against an epitope on the $\alpha$ chain polypeptide $\left(\alpha_{\mathrm{m}}\right.$ or $\left.\alpha_{1}\right)(7,9,10)$ of the CD11b (MAC-1, Mo1) heterodimer (7). Both MoAb 60.3 and 60.1 have been shown to inhibit neutrophil adhesive functions in response to some agonists $(7,10)$. MoAb 60.5 is an IgG2a antibody directed against a class I HLA antigen found on human peripheral blood leukocytes and on EC $(6,8)$; it was used as a control antibody. In experiments with each of these purified antibodies we pretreated neutrophils with saturating concentrations of the $\mathrm{MoAb}$ for $10 \mathrm{~min}$ at room temperature before addition of the suspensions to EC monolayers. The antibody was present in the PMN suspensions throughout their incubation with the EC.

Monoclonal antibody Mol $\alpha$, a murine IgG2a directed against an epitope on the $\alpha_{m}$ chain of the CD1 1b (Mo1, MAC-1) heterodimer (10, 15), was generously provided by R. F. Todd III, (University of Michigan Medical Center, Ann Arbor, MI) in mouse ascites. Mouse ascites fluid containing an IgG2a MoAb directed against a human HLA-B27 antigen (Cooper Biomedical, Inc., Malvern, PA) or against a 200,000 mol wt human leukocyte antigen (GAP 8.3, American Type Culture Collection, provided by Charles Parker, University of Utah) were used as control antibodies. MoAb Mol $\alpha$ or the control MoAb was incubated with PMN suspensions for $20 \mathrm{~min}$ at room temperature before addition to EC and was present throughout the incubation. Preliminary experiments indicated that MoAb Mol $\alpha$ inhibited fMLP-stimulated PMN adherence to cell-free surfaces and to EC in a concentration-related fashion with a maximal effect at a dilution of the original ascites fluid of $1: 1,000$.

The percent inhibition of agonist-stimulated PMN adherence by MoAbs was calculated after subtracting the value for basal, unstimulated PMN adherence from the values for agonist-stimulated adherence in the presence of MoAb or control buffer. Reduction of stimulated adherence by MoAb to values equal to, or lower than, unstimulated adherence is reported as $100 \%$ inhibition.

\section{Results}

Effect of monoclonal antibodies on adherence stimulated by agonists that directly increase neutrophil adhesiveness. MoAb 60.3 identifies an antigen that is broadly expressed on myeloid and lymphoid leukocytes including PMNs (10,14). It inhibits a number of neutrophil functions that are dependent on adhesion $(7,8)$. Therefore, we evaluated the ability of MoAb 60.3 to inhibit PMN adherence in response to soluble stimuli that directly enhance the adhesiveness of neutrophils. In preliminary experiments we found that MoAb 60.3 inhibited fMLPstimulated PMN adherence to EC monolayers and to albumin-treated petri dishes in a concentration-dependent fashion with maximal inhibition at $10 \mu \mathrm{g} / \mathrm{ml}$. This result is similar to the concentration dependence previously reported by Harlan et al., using other agonists (16). A time course (1-30 min) demonstrated that maximal inhibition occurred when PMN suspensions were pretreated with MoAb $60.3(10 \mu \mathrm{g} / \mathrm{ml})$ for as little as $5 \mathrm{~min}$ at $22^{\circ} \mathrm{C}$ before stimulation with fMLP. In eight subsequent experiments in which PMNs were pretreated with MoAb 60.3 and their adherence to EC in response to fMLP was measured, the mean inhibition was $98 \%$ (Table I and Fig. 1). In several of these experiments the adherence of fMLPstimulated PMNs was reduced below the level of basal, unstimulated adherence in the presence of MoAb 60.3. We then examined the effect of the antibody on adherence stimulated by other soluble agonists added to neutrophil suspensions in the fluid phase, using concentrations of the agonists that caused maximal or near-maximal adhesion. MoAb 60.3 inhibited adherence to EC in response to $\mathrm{LTB}_{4}, \mathrm{PAF}$, TNF, PMA, and calcium ionophore $\mathrm{A} 23187$ by $93-100 \%$ under these conditions (Table I). The antibody also potently inhibited PMN aggregation stimulated by fMLP, LTB 4 , PAF, C5a fragments in zymosan-activated plasma, and PMA (not shown), and inhibited neutrophil adherence to cell-free surfaces (Table I). In

Table I. Inhibition of Neutrophil Adherence by Monoclonal Antibodies

\begin{tabular}{|c|c|c|c|c|}
\hline Agonist & MoAb 60.3 & MoAb 60.1 & MoAb 60.5 & Mol $\alpha$ \\
\hline & $\%$ & $\%$ & $\%$ & $\%$ \\
\hline \multicolumn{5}{|c|}{ Adherence to human EC: } \\
\hline fMLP & $98 \pm 4(8)$ & $70 \pm 9(2)$ & $16 \pm 12(5)$ & $54(1)$ \\
\hline $\mathrm{LTB}_{4}$ & $100 \pm 1(4)$ & $83(1)$ & $18 \pm 15(2)$ & - \\
\hline PAF & $98 \pm 11(4)$ & - & $10(1)$ & - \\
\hline $\mathrm{TNF} \alpha$ & $100 \pm 0(4)$ & 一 & - & - \\
\hline PMA & $100 \pm 1(2)$ & $79 \pm 12(3)$ & $0(1)$ & $68 \pm 10(2)$ \\
\hline Io $\mathrm{A} 23187$ & $95 \pm 7(2)$ & - & - & 一 \\
\hline \multicolumn{5}{|c|}{ Adherence to cell-free surfaces: } \\
\hline fMLP & $89 \pm 9(5)$ & $86 \pm 10(4)$ & $9(1)$ & $50 \pm 15(4)$ \\
\hline $\mathrm{LTB}_{4}$ & $99 \pm 2(2)$ & $99 \pm 2(2)$ & - & - \\
\hline PAF & $97(1)$ & $91 \pm 6(2)$ & $20(1)$ & - \\
\hline
\end{tabular}

In each experiment neutrophil suspensions that had been preincubated with MoAb $(60.3-10 \mu \mathrm{g} / \mathrm{ml} ; 60.1-40 \mu \mathrm{g} / \mathrm{ml} ; 60.5-10$ or $40 \mu \mathrm{g} / \mathrm{ml}$; Mol $\alpha-1: 1,000$ ) or control solution (PBS) were added to EC monolayers or to cell-free surfaces, an agonist or control buffer was added, and the suspensions were incubated as described in Methods. The concentrations and durations of incubation for each agonist were: $\mathrm{fMLP}, \mathrm{LTB}_{4}, \mathrm{PAF}, 1 \mu \mathrm{M}, 5 \mathrm{~min}$; PMA, 10 or 100 $\mathrm{ng} / \mathrm{ml}, 30 \mathrm{~min}$; Io A23187, $10 \mu \mathrm{M}, 5$ or $30 \mathrm{~min}$; TNF $\alpha, 1,000$ or $10,000 \mathrm{U} / \mathrm{ml}, 30 \mathrm{~min}$. Each of the agonists listed stimulated PMN adherence to cell-free surfaces in addition to EC, but MoAb inhibition of this response was studied with only $\mathrm{fMLP} \mathrm{LTB}_{4}$, and PAF. The mean $( \pm \mathrm{SD})$ percent inhibition of adherence resulting from treatment with MoAb, calculated as described in Methods, is shown with the number of experiments in parentheses. 


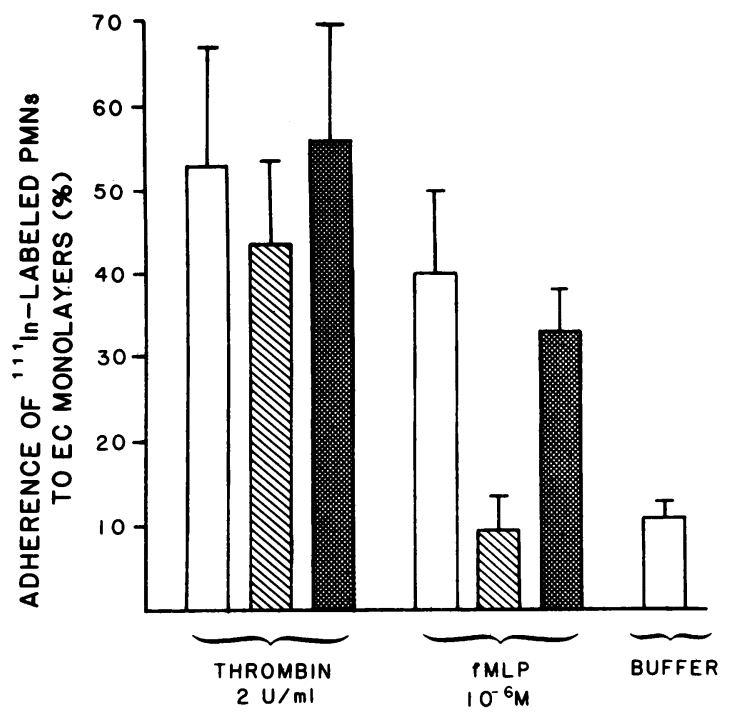

Figure 1. MoAb 60.3 minimally inhibits neutrophil adherence to thrombin-stimulated EC. Neutrophil suspensions were preincubated with PBS (open bars), MoAb $60.3(10 \mu \mathrm{g} / \mathrm{ml}$, hatched bars), or MoAb $60.5(10 \mu \mathrm{g} / \mathrm{ml}$, stippled bars), added to EC monolayers, thrombin, fMLP, or control buffer was added, and adherence was determined after a 5-min incubation. In one experiment EC were pretreated with thrombin or control buffer for $5 \mathrm{~min}$ and washed (Methods) before addition of the neutrophil suspensions. Each bar indicates the mean +1 SD of four (thrombin) or eight experiments (fMLP and control buffer).

contrast, MoAb 60.5 caused a maximum of $20 \%$ inhibition of PMN adherence in response to various agonists (Table I).

Effect of MoAb 60.3 on EC-dependent neutrophil adherence stimulated by thrombin. Because MoAb 60.3 potently inhibited neutrophil adherence to EC stimulated by a variety of agonists that directly enhance PMN adhesiveness (Table I), we examined its effect on adherence stimulated by thrombin. Human $\alpha$-thrombin induces increased neutrophil adhesion by an endothelial cell-dependent mechanism, and does not directly alter neutrophil adhesiveness (4). In four experiments, MoAb 60.3 caused a mean $18 \%$ inhibition of the adherence stimulated by $2 \mathrm{U} / \mathrm{ml}$ thrombin, whereas it completely inhibited fMLP-stimulated adherence in parallel incubations (Fig. 1). Thrombin-stimulated adherence was unchanged or slightly enhanced by MoAb 60.5. We found similar results when endothelial cells were preincubated with thrombin $\left(5 \mathrm{~min}, 37^{\circ} \mathrm{C}\right)$ followed by washing and the addition of control or MoAbtreated PMNs, or when the thrombin was added to MoAbpretreated neutrophil suspensions in the presence of endothelial cells (Methods). Increasing the concentration of MoAb 60.3 from 10 to $40 \mu \mathrm{g} / \mathrm{ml}$ did not further increase the inhibition.

The experiments in Fig. 1 demonstrate that MoAb 60.3 had a minor effect on PMN adherence caused by concentrations of thrombin that induce maximal EC-dependent adhesion $(4,13)$, suggesting that the PMN CDw 18 complex plays a minor role and is not absolutely required for neutrophil adherence to thrombin-stimulated endothelium under these conditions. To determine if the neutrophil CDw18 glycoproteins are required for submaximal endothelial cell-dependent adhesion, we examined the effect of MoAb 60.3 on neutrophil adherence stimulated by lower concentrations of thrombin. In two ex- periments MoAb 60.3 reduced PMN adherence induced by submaximal concentrations of thrombin $(0.1-0.5 \mathrm{U} / \mathrm{ml})$ by $22-40 \%$. This result suggests that the neutrophil glycoproteins play a greater role in the adhesive interaction when EC are activated under submaximal conditions. However, the adherence of MoAb 60.3-treated PMNs remained significantly elevated at suboptimal concentrations of thrombin and was not reduced to basal levels at any thrombin concentration (Fig. 2).

Effect of MoAb 60.3 on EC-dependent neutrophil adherence stimulated by $L T C_{4}$. We then examined the effect of MoAb 60.3 on neutrophil adherence induced by $\mathrm{LTC}_{4}$, an agonist that also acts by an endothelial cell-dependent mechanism (12). The $\mathrm{LTC}_{4}$-stimulated adhesiveness of the endothelial cells is both concentration- and time-dependent (12); in contrast to thrombin, which causes maximal adherence at 5-10 $\min (4), \mathrm{LTC}_{4}$-induced neutrophil adherence continuously increases during this period, with a peak at $30 \mathrm{~min}$ (12). Therefore we studied the ability of MoAb 60.3 to inhibit submaximal (at $5 \mathrm{~min}$ ) and maximal (at $30 \mathrm{~min}$ ) $\mathrm{LTC}_{4}$-induced adherence. At $5 \mathrm{~min}$, the adherence of PMNs treated with MoAb $60.3(10 \mu \mathrm{g} / \mathrm{ml})$ was $15 \pm 10 \%$ compared to $22 \pm 7 \%$ for PMNs treated with control buffer $(n=4, P=0.05$ by paired $t$ test), representing a mean $65 \%$ inhibition (range $9-100 \%$ ) (Figs. $3 A$, 4). At $30 \mathrm{~min}$ the adherence of MoAb 60.3-treated PMNs was $35 \pm 11 \%$ versus $42 \pm 15 \%$ for control neutrophils $(n=7, P$ $=0.04$ by paired $t$ test), a mean $21 \%$ inhibition (range 3-36\%). In 2 of these experiments there was essentially no inhibition of $\mathrm{LTC}_{4}$-induced adherence by MoAb 60.3 at $30 \mathrm{~min}$ (5\% or less; Fig. 4), although the antibody inhibited adherence stimulated by one or more of the agonists shown in Table $I$ in parallel incubations (Figs. 3 and 4). In two additional experiments the adherence of MoAb 60.3-treated PMNs was $70-100 \%$ of the adherence of control PMNs to EC stimulated with submaximal or maximal concentrations of $\mathrm{LTC}_{4}\left(0.1-5 \times 10^{-6} \mathrm{M}\right)$

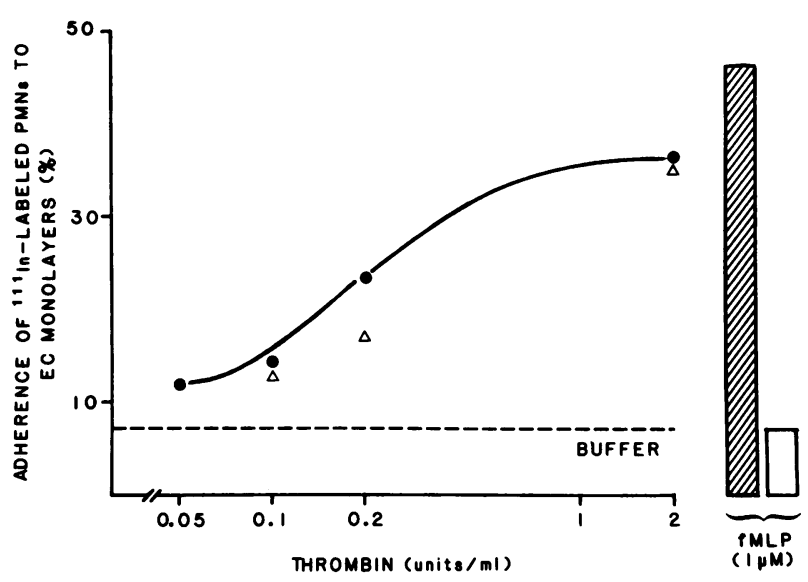

Figure 2. Neutrophils treated with MoAb 60.3 adhere to endothelial cells stimulated with submaximal as well as maximal concentrations of thrombin. PMNs were preincubated with control buffer (closed circles) or MoAb 60.3 (open triangles), added to EC monolayers, thrombin was added in the indicated concentrations, and adherence was determined after a 5-min incubation as described in Methods and Fig. 1. The adherence of neutrophils pretreated with control buffer (hatched bar) or MoAb 60.3 (open bar) in response to fMLP was measured in parallel. The dashed line indicates PMN adherence in response to control buffer in place of thrombin or fMLP. The data from one experiment are shown; a second experiment yielded qualitatively similar results. 

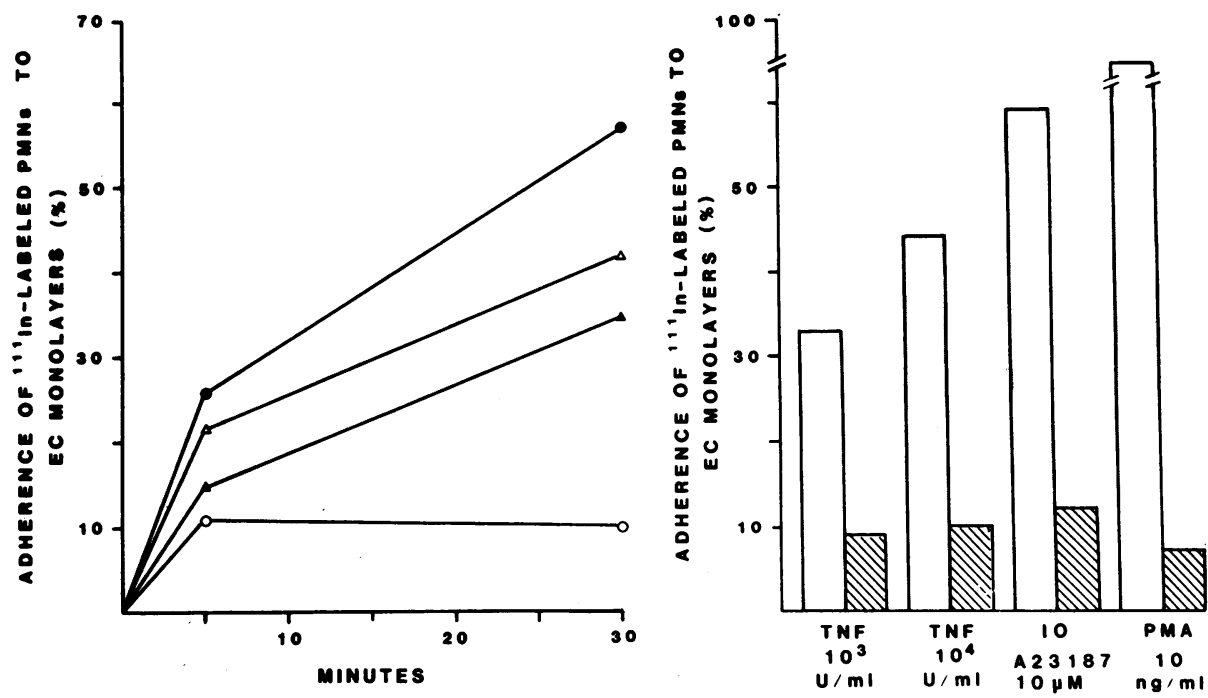

Figure 3. (A) Effect of MoAb 60.3 and 60.5 on $\mathrm{LTC}_{4}$-stimulated neutrophil adherence to EC. PMNs were pretreated with MoAbs 60.3 (closed triangles), 60.5 (closed circles) or PBS (open triangles) and were then incubated with $\mathrm{EC}$ in the presence of $\mathrm{LTC}_{4}(1 \mu \mathrm{M})$ for the indicated times; in some experiments the PMNs were incubated for 5 min with EC that had been pretreated with $\mathrm{LTC}_{4}$ for the indicated time periods. The concentrations of $\mathrm{MoAb} 60.3$ and 60.5 were $10 \mu \mathrm{g} / \mathrm{ml}$ in each experiment. The open circles indicate the adherence of control PMNs (no MoAb or $\mathrm{LTC}_{4}$ ). Each point indicates the mean of a minimum of 4 experiments (4-7) with the exception of the closed circles, which indicate 2 experiments. The standard deviations

were: 5 min, open circle- $2 \%$, open triangle-7\%, closed triangle- $10 \%$, closed circle- $10 \%$; 30 minutes, open circle- $2 \%$, open triangle- $15 \%$, closed triangle-11\%, closed circle-11\%. (B) MoAb 60.3 potently inhibits neutrophil adherence induced by TNF, IoA23187, and PMA. PMN suspensions were pretreated with MoAb $60.3(10 \mu \mathrm{g} / \mathrm{ml}$, hatched bars) or PBS (open bars) for $10 \mathrm{~min}$, added to EC monolayers, an agonist (shown on the horizontal axis) was added, and adherence was determined after a 30-min incubation. Each pair of bars indicates the result of one experiment. Additional experiments confirmed that MoAb 60.3 inhibited neutrophil adherence stimulated by each of the agonists (Table I).

for $30 \mathrm{~min}$. The results were similar when control and MoAb 60.3-treated neutrophils were added to EC that had been preincubated with $\mathrm{LTC}_{4}$ for the indicated times and when neutrophil suspensions were added to the EC monolayers and incubated for the specified times after addition of $\mathrm{LTC}_{4}$. Increasing the concentration of the antibody to $40 \mu \mathrm{g} / \mathrm{ml}$ did not further increase the inhibition after a 30 -min incubation $\left(10^{-6}\right.$ $\left.\mathrm{M} \mathrm{LTC}_{4} ; n=2\right)$. Pretreatment of the neutrophils with MoAb 60.5 resulted in adherence that was the same as, or greater than, the adherence of control leukocytes (Fig. $3 A$ ).

To exclude the possibility that the activity of MoAb 60.3 decreased during the longer 30-min incubation periods (Figs. 3 $A$ and 4), we measured its effect on PMA-, ionophore-, and
TNF-induced adherence in parallel incubations of this duration. The antibody completely inhibited PMN adherence in response to these mediators at $30 \mathrm{~min}$, including the dramatic increase in adhesion stimulated by PMA (Fig. $3 \mathrm{~B}$ ).

Effect of monoclonal antibodies directed at other epitopes of CDw18 on neutrophil adherence. MoAb $60.1(40 \mu \mathrm{g} / \mathrm{ml})$ inhibited fMLP-, LTB $_{4}$-, PAF-, and PMA-stimulated PMN adherence to EC and to cell-free surfaces by $70-99 \%$ (Table I). In contrast, in three experiments the antibody inhibited $\mathrm{LTC}_{4}$ stimulated adherence $\left(10^{-6} \mathrm{M}, 30 \mathrm{~min}\right)$ by $29 \pm 19 \%$ (range 8-43\%). In three additional experiments MoAb 60.1 inhibited thrombin-stimulated adherence $(2 \mathrm{U} / \mathrm{ml}, 5 \mathrm{~min})$ by $33 \%$ or less.

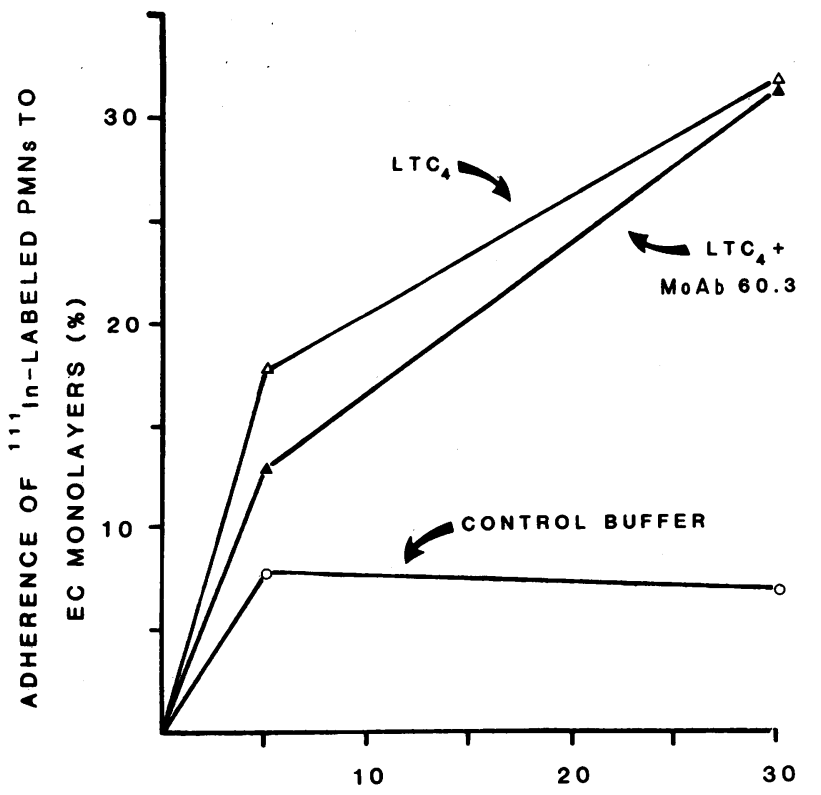

TIME (MINUTES)

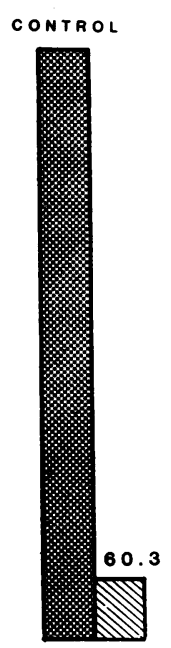

fMLP
Figure 4. Neutrophils treated with MoAb 60.3 avidly adhere to $\mathrm{LTC}_{4}$-stimulated endothelial cells. PMN suspensions were pretreated with MoAb 60.3 (10 $\mu \mathrm{g} / \mathrm{ml}$ ) or PBS for $10 \mathrm{~min}$, added to EC monolayers, $\mathrm{LTC}_{4}\left(10^{-6} \mathrm{M}\right)$ was added, and the incubations were stopped at the indicated times. The adherence of PMNs pretreated with control buffer or MoAb 60.3 in response to fMLP was measured after a 5-min incubation in parallel. The figure demonstrates the data from one experiment. 
In two experiments MoAb Mol $\alpha$ inhibited PMA-induced neutrophil adherence to EC by $61-75 \%$ whereas ascites fluids containing control antibody did not inhibit adherence. In parallel incubations in these experiments the adherence of MoAb Mol $\alpha$-treated neutrophils was the same as, or greater than, that of control antibody-treated neutrophils in response to thrombin $(2 \mathrm{U} / \mathrm{ml}, 5 \mathrm{~min})$ or $\mathrm{LTC}_{4}\left(10^{-6} \mathrm{M}, 30 \mathrm{~min}\right)$.

\section{Discussion}

Identification of the molecular mechanisms involved in neutrophil adherence to endothelium promises to be a key to deciphering processes that control acute inflammation, contribute to inflammatory vascular injury, and that are disrupted in certain life-threatening syndromes of leukocyte dysfunction (17). Recent observations indicate that the CD11b (MAC-1, Mo1) heterodimer of the CDw 18 glycoprotein complex is critically involved in neutrophil adhesive interactions including aggregation, adhesion to nonbiologic surfaces, and adhesion to endothelium (6-11). In this study we found that a monoclonal antibody directed against a common determinant on the glycoproteins of the leukocyte CDw18 complex, MoAb 60.3, and antibodies directed against epitopes on the $\alpha$ polypeptide chain of the CD1 $1 \mathrm{~b}$ heterodimer, MoAbs 60.1 and Mol $\alpha$, inhibited neutrophil adherence to endothelium and to cell-free surfaces stimulated by a variety of agonists of different classes (Table I). The relative potency of the antibodies as inhibitors of PMN adherence to EC (MoAb $60.3>60.1 \geq \operatorname{Mol} \alpha$, Table I) was similar to the hierarchy of inhibition of leukocyte adhesiondependent functions by other MoAbs directed against specific epitopes on the $\alpha$ or $\beta$ polypeptides (18). The results in Table I demonstrate that one mechanism for rapid adhesion of PMNs to endothelium induced by these agonists in the fluid phase involves activation of the neutrophils and expression of the adhesive glycoprotein. For some of the agonists, particularly fMLP and $\mathrm{LTB}_{4}(3,19)$, the relative contributions of a direct effect on the neutrophils and an endothelial cell-dependent mechanism has been previously questioned $(1-4,19,20)$. Human TNF $\alpha$ causes both a direct enhancement of neutrophil adhesiveness as well as increased neutrophil adherence resulting from an alteration in the EC surface, depending on the time of incubation $(6,8)$. The rapid ( $30 \mathrm{~min})$, direct increase in PMN adhesiveness induced by TNF $\alpha$ is reported to be completely inhibited by treatment with MoAbs 60.3 and 60.1 (6), an observation that is confirmed by the data in Table I. The findings in Table I are also consistent with previously published reports of the effects of MoAbs 60.3 and 60.1 on PMN adherence induced by PMA, Io A23187, and FMLP under different temporal conditions $(7,16)$ and with observations that anti-Mol $\alpha$ inhibits neutrophil aggregation induced by fMLP and C5a (10).

MoAb 60.3 was a particularly useful reagent because of its potency and because it is directed against an epitope found on each of the heterodimeric members of the CDw18 complex and therefore against the GP 150,95 as well as the CD11b (Mo1, MAC-1) heterodimers $(1,7,10)$. Both of these glycoproteins are expressed on the surface of activated PMNs and may be important in their adhesive responses $(7,16,18,21)$. Furthermore, treatment of normal PMNs with MoAb 60.3 reproduces the adherence-dependent functional deficiencies found in granulocytes from patients that are genetically deficient in the CDw 18 molecules $(7,16)$. Therefore, we examined the effects of MoAb 60.3 on endothelial cell-dependent PMN adherence stimulated by thrombin and $\mathrm{LTC}_{4}$ in detail, and in selected experiments compared them to the effects of monoclonal antibodies directed against other epitopes on the CDw18 glycoproteins. In each experiment we did parallel incubations using fMLP or another agonist that directly enhanced the adhesiveness of the neutrophils (Table I) to document the efficacy of the anti-CDw18 antibodies.

In contrast to adherence stimulated by the agonists in Table I, the endothelial cell-dependent neutrophil adherence induced by peak concentrations of thrombin or $\mathrm{LTC}_{4}$ was only minimally altered by saturating concentrations of antibodies directed against epitopes of the CDw 18 glycoprotein complex. MoAb 60.3, which inhibited neutrophil adherence induced by fMLP and other direct PMN agonists by $95-100 \%$ (Table I), inhibited maximal thrombin- and $\mathrm{LTC}_{4}$-stimulated adherence by approximately $20 \%$ (Results and Figs. 1-4). The adherence of PMNs to EC that were treated with submaximal concentrations of thrombin was inhibited to a greater extent by MoAb 60.3 (Results), as was adherence to $\mathrm{LTC}_{4}$-stimulated endothelial cells at a submaximal time point (Figs. $3 A$ and 4; 5 min time point). This suggests that expression of the neutrophil glycoprotein complex may amplify the adhesive interaction under conditions in which the EC are not maximally stimulated. We previously observed that a fraction of the PMNs adherent to thrombin- and $\mathrm{LTC}_{4}$-stimulated endothelium were polarized $(4,12,13)$, indicating that they had been activated. These leukocytes likely express increased numbers of CDw1 18 heterodimers on their surfaces $(10,16)$, an event predicted to potentiate adhesion and to be inhibited by MoAb 60.3.

Although the neutrophil glycoproteins may play a greater role under conditions in which submaximal endothelial celldependent adherence occurs, they are apparently not required for significantly-enhanced adherence by the EC-dependent mechanism. This observation is clearly demonstrated in Figs. 1,2 , and 4 (30' time point). Furthermore, in some experiments there was essentially no inhibition of thrombin- or $\mathrm{LTC}_{4}$-induced adhesion, although there was complete inhibition of adhesion stimulated by fMLP or PMA in parallel, "side-byside" incubations (Figs. 2 and 4). These findings support previous observations suggesting that there are molecular changes in the EC surface in response to thrombin and $\mathrm{LTC}_{4}$ that promote neutrophil adherence $(4,12)$, and indicate that the neutrophil CDw18 glycoprotein complex is not absolutely required for this adhesive interaction. In view of the redundant mechanisms involved in inflammation, it is not unexpected that more than one molecular event may be involved in a fundamental response such as granulocyte adhesion. ${ }^{2}$

In contrast to our findings, Pohlman et al. concluded that endothelial cell-dependent neutrophil adherence induced by interleukin 1, TNF $\alpha$, and lipopolysaccharide occurs by a mechanism that is dependent on the CDw18 molecules (8). This discrepancy may be due to the fact that there are signifi-

2. A recent report suggests that murine neutrophils have a surface protein that is distinct from the CDw 18 glycoproteins and that participates in PMN adhesion to endothelium. (Lewinsohn, D. M., R. F. Bargatze and E. C. Butcher. 1987. Leukocyte-endothelial cell recognition: evidence of a common molecular mechanism shared by neutrophils, lymphocytes, and other leukocytes. J. Immunol. 138:43134321). 
cant differences in the endothelial cell-dependent neutrophil adhesion induced by these cytokines $(5,6,8)$ compared to that induced by thrombin and $\operatorname{LTC}_{4}(4,12,13)$, and that different molecular mechanisms are involved. However Pohlman et al. also noted that PMNs treated with MoAb 60.3, and neutrophils from a CDw18-deficient patient, adhered to TNF-, interleukin 1-, and endotoxin-treated EC, albeit much less avidly than did control leukocytes (8). This observation is consistent with the hypothesis that one or more proadhesive molecules on the endothelial surface mediate the interaction with PMNs without an absolute requirement for the neutrophil glycoproteins. The identity of the endothelial cell adhesive molecule(s) remains to be determined. We previously suggested that PAF that is endogenously synthesized by thrombin- or $\mathrm{LTC}_{4}$-activated $\mathrm{EC}$ may directly mediate the adhesive interaction $(4,12$, 13). Taken at face value, the experiments in Table I argue against this hypothesis since MoAb 60.3 inhibited PMN adherence induced by exogenous PAF but had little effect on maximal adherence stimulated by thrombin or $\mathrm{LTC}_{4}$ (Figs. 1-4). However, it is possible that PAF associated with a biologic surface (i.e. the plasma membrane of the EC) interacts with the PMN in a different way from micellar PAF presented in solution. In the first condition the PAF will likely interact with a localized part of the neutrophil plasma membrane in vectorial fashion, whereas in the second condition there will be global presentation of the PAF molecules to the PMN surface. The mechanisms involved in the adhesive interaction may vary in the two situations. In addition, the motion and redistribution of receptors in the neutrophil plasma membrane (22) may vary when PMN adhesion occurs in one condition as opposed to the other; neutrophil receptor mobility may also vary when PMNs adhere to the altered surface of an activated endothelial cell, rather than a quiescent EC, since the nature of the surface influences these events $(22,23)$. We cannot exclude the possibility there is localized expression of the CDw18 glycoproteins on an area of the PMN membrane adjacent to the EC when the neutrophils are activated by PAF associated with the endothelial surface and that the anti-CDw18 antibodies were excluded from this microenvironment in our experiments. Finally, it is also possible that the MoAb 60.3-inhibitable PMN adherence to EC stimulated by thrombin and $\mathrm{LTC}_{4}$ (20-65\%, depending on whether the EC are maximally or submaximally activated; Results) is mediated by endogenously synthesized PAF, whereas the remainder is mediated by a second molecular species (4).

Our observations also suggest that the neutrophil CDw18 glycoprotein molecules may have important functions aside from mediating adhesion. Neutrophils from patients with a genetic deficiency of the CDw18 complex cannot migrate to extravascular sites in vivo (7-11). Treatment of normal PMNs with MoAb 60.3 reproduces the deficits of adhesive function found in leukocytes from CDw18-deficient patients, including the chemotactic defect $(7,16)$. Yet MoAb 60.3-treated PMNs can partially or fully adhere to EC induced to become adhesive by thrombin, $\mathrm{LTC}_{4}$, or other mediators (Figs. 1-3 and Ref. 8), and neutrophils from patients with $\mathrm{CDw} 18$ deficiency retained the ability to adhere to EC in a basal, unstimulated fashion (16, 24) or the partial ability to undergo enhanced adherence by an EC-dependent mechanism (8). Similar events may occur in vivo since neutrophils accumulate in microvessels adjacent to sites of infection but do not migrate into extravascular tissues in some patients with CDw18 deficiency (11). These observa- tions suggest that adhesion is required, but not sufficient, for directed PMN migration, further suggesting that CDw18 deficiency results in additional defects in chemotaxis and other complex, adhesion-related, neutrophil functions. The glycoproteins may be important in transducing signals from the external environment to intracellular regulatory systems. This possibility is suggested by observations that other surface glycoproteins that are important in adhesion-related functions have extensive intracellular domains providing sites for interaction with cytoplasmic proteins and cytoskeletal elements (25) and that key regulatory proteins are concentrated at points of cell-cell adhesion in some mammalian cells (26).

\section{Acknowledgments}

The authors are grateful to Patrick Beatty, George Broze, John Fenton, John Harlan, Charles Parker, Joshua Rokach, Michael Shepard, and Robert F. Todd for generously providing biologic materials, Donelle Benson and Patricia Cain for technical assistance, Leona Archuleta and Linda Jara for preparation of the manuscript and figures, and John Harlan, Joseph Sisson, and Stephen M. Prescott for helpful discussions. We thank Steve Prescott and John Harlan for review of the manuscript.

Supported by grants from the Nora Eccles Treadwell Foundation and the U. S. Public Health Service (R01 HL-35828), and a Grant in Aid (84-975) and an Established Investigator Award (85-204) from the American Heart Association.

\section{References}

1. Harlan, J. M., B. R. Schwartz, W. J. Wallis, and T. H. Pohlman. 1987. The role of neutrophil membrane proteins in neutrophil emigration. In Leukocyte Emigration and its Sequelae. H. Z. Movat, editor. S. Karger AG, Basel. 94-104.

2. Zimmerman, G. A., T. M. McIntyre, and S. M. Prescott. 1987. Naturally occurring lipids influence the interaction of human endothelial cells and neutrophils. In Leukocyte Emigration and its Sequelae. H. Z. Movat, editor. S. Karger AG, Basel. 105-118.

3. Tonnesen, M. G., L. A. Smedley, and P. M. Henson. 1984. Neutrophil-endothelial cell interactions. Modulation of neutrophil adhesiveness induced by complement fragments $\mathrm{C5a}$ and $\mathrm{C5a}$ desarg and formyl-methionyl-leucyl-phenylalanine in vitro. J. Clin. Invest. 74:1581-1592.

4. Zimmerman, G. A., T. M. McIntyre, and S. M. Prescott. 1985. Thrombin stimulates the adherence of neutrophils to human endothelial cells in vitro. J. Clin. Invest. 76:2235-2246.

5. Bevilacqua, M. P., J. S. Pober, M. E. Wheeler, R. S. Cotran, and M. A. Gimbrone. 1985. Interleukin-1 acts on cultured human vascular endothelium to increase the adhesion of polymorphonuclear leukocytes, monocytes, and related leukocyte cell lines. J. Clin. Invest. 76:2003-2011.

6. Gamble, J. R., J. M. Harlan, S. J. Klebanoff, and M. A. Vadas. 1985. Stimulation of the adherence of neutrophils to umbilical vein endothelium by human recombinant tumor necrosis factor. Proc. Natl. Acad. Sci. USA. 82:8667-8671.

7. Wallis, W. J., D. D. Hickstein, B. R. Schwartz, C. H. June, H. D. Ochs, P. G. Beatty, S. J. Klebanoff, and J. M. Harlan. 1986. Monoclonal antibody-defined functional epitopes on the adhesion-promoting glycoprotein complex (CDw18) of human neutrophils. Blood. 67:1007-1013.

8. Pohlman, T. H., K. A. Stanness, P. G. Beatty, H. D. Ochs, and J. M. Harlan. 1986. An endothelial cell surface factor(s) induced in vitro by lipopolysaccharide, interleukin-1, and tumor necrosis factor- $\alpha$ increases neutrophil adherence by a CDw18-dependent mechanism. $J$. Immunol. 136:4548-4553. 
9. Springer, T. A. 1985. The LFA-1, MAC-1 glycoprotein family and its deficiency in an inherited disease. Fed. Proc. 44:2660-2663.

10. Todd, R. F., III, and M. A. Arnaout. 1986. Monoclonal antibodies that identify Mol and LFA-1, two human leukocyte membrane glycoproteins: A review. In Reinherz, E. L., B. F. Haynes, L. M. Nadler, and I. D. Bernstein. Leukocyte Typing II: Volume 3. Human myeloid and hematopoeic cells. Springer-Verlag, New York. 95-108.

11. Anderson, D. C., F. C. Schmalsteig, M. J. Finegold, B. J. Hughes, R. Rothlein, L. J. Miller, S. Kohl, M. F. Tosi, R. L. Jacobs, T. C. Waldrop, A. S. Golman, W. T. Shearer, and T. A. Springer. 1985. The severe and moderate phenotypes of heritable MAC-1, LFA-1 deficiency: Their quantitative definition and relation to leukocyte dysfunction and clinical features. J. Infect. Dis. 152:668-689.

12. McIntyre, T. M., G. A. Zimmerman, and S. M. Prescott. 1986. Leukotrienes $C_{4}$ and $D_{4}$ stimulate human endothelial cells to synthesize platelet-activating factor and bind neutrophils. Proc. Natl. Acad. Sci. USA. 83:2204-2208.

13. Zimmerman, G. A., T. M. McIntyre, and S. M. Prescott. 1986. Thrombin stimulates neutrophil adherence by an endothelial cell-dependent mechanism: characterization of the response and relationship to platelet-activating factor synthesis. Ann. NY Acad. Sci. 485:349367.

14. Beatty, P. G., J. A. Ledbetter, P. J. Martin, T. H. Price, and J. A. Hansen. 1983. Definition of a common leukocyte cell-surface antigen (Lp95-150) associated with diverse cell-mediated immune functions. J. Immunol. 131:2913-2918.

15. Arnaout, M. A., R. F. Todd, III, N. Dana, J. Melamed, S. F. Schlossman, and H. R. Colten. 1983. Inhibition of phagocytosis of complement C3- or immunoglobulin G-coated particles and of C3bi binding by monoclonal antibodies to a monocyte-granulocyte membrane glycoprotein (Mo1). J. Clin. Invest. 72:171-179.

16. Harlan, J. M., P. D. Killen, F. M. Senecal, B. R. Schwartz, E. K. Yee, R. F. Taylor, P. G. Beatty, T. H. Price, and H. D. Ochs. 1985. The role of neutrophil membrane glycoprotein GP-150 in neutrophil adherence to endothelium in vitro. Blood. 66:167-178.

17. Gallin, J. I. 1985. Leukocyte adherence-related glycoproteins LFA-1, Mo1, and p150,95. A new group of monoclonal antibodies, a new disease, and a possible opportunity to understand the molecular basis of leukocyte adherence. J. Infect. Dis. 152:661-664.

18. Springer, T. A., L. J. Miller, and D. C. Anderson. 1986. p150,95, the third member of the MAC-1, LFA-1 human leukocyte adhesion glycoprotein family. J. Immunol. 136:240-245.

19. Hoover, R. L., M. J. Karnovsky, K. F. Austen, E. J. Corey, and R. A. Lewis. 1984. Leukotriene $B_{4}$ action on endothelium mediates augmented neutrophil/endothelial adhesion. Proc. Natl. Acad. Sci. USA. 82:2191-2193.

20. Schleimer, R. P., and B. K. Rutledge. 1986. Cultured human vascular endothelial cells acquire adhesiveness for neutrophils after stimulation with interleukin 1, endotoxin, and tumor-promoting phorbol diesters. J. Immunol. 136:15:649-654.

21. Anderson, D. C., L. J. Miller, F. C. Schmalstieg, R. Rothlein, and T. A. Springer. 1986. Contributions of the MAC-1 glycoprotein family to adherence-dependent granulocyte functions: structure-function assessments employing subunit-specific monoclonal antibodies. $J$. Immunol. 137:15-27.

22. Wilkinson, P. C. 1987. Leukocyte locomotion and accumulation: the contributions of cell polarity and cell growth. In Leukocyte Emigration and its Sequelae. H. Z. Movat, editor. S. Karger AG, Basel. $1-13$.

23. Hafeman, D. G., L. M. Smith, D. T. Fearon, and H. M. McConnell. 1982. Lipid monolayer-coated solid surfaces do not perturb the lateral motion and distribution of $\mathrm{C} 3 \mathrm{~b}$ receptors on neutrophils. J. Cell Biol. 94:224-227.

24. Buchanan, M. R., C. A. Crowly, R. E. Rosin, M. A. Gimbrone, and B. M. Babior. 1982. Studies on the interaction between GP-180deficient neutrophils and vascular endothelium. Blood. 60:160-165.

25. Hemperly, J. J., B. A. Murray, G. M. Edelman, and B. A. Cunningham. 1986. Sequence of a cDNA clone encoding the polysialic acid-rich and cytoplasmic domains of the neural cell adhesion molecule N-CAM. Proc. Natl. Acad. Sci. USA. 83:3037-3041.

26. Maher, P. A., E. B. Pasquale, J. Y. J. Wang, and S. J. Singer. 1985. Phosphotyrosine-containing proteins are concentrated in focal adhesions and intercellular junctions in normal cells. Proc. Natl. Acad. Sci. USA. 82:6576-6580. 